\title{
Shortening of a Raman fiber laser by inserting ytterbium doped
} fiber

\author{
E.B. Mejía, L. De la Cruz May, and D.V. Talavera \\ ${ }^{1}$ (Centro de Investigaciones en Óptica, Loma del bosque 115, 37150 León, Gto., México.) \\ ${ }_{2}^{2}$ (Universidad Autónoma del Carmen, Facultad de Ingeniería, C. 56, No. 4. 24180, Cd. del Carmen, Campeche, \\ México) \\ ${ }_{3}^{3}$ (Universidad Politécnica de Guanajuato, Av. Universidad Norte S/N, 38483 Cortázar, Gto., México.)
}

\begin{abstract}
Once reached the threshold for operation, Raman fiber lasers with high output couplers convert the pump signal into stokes signals very efficiently. However, high threshold implies lower overall efficiency and hence the ratio slope efficiency-to-threshold power (i.e., the figure of merit) is low. By maintaining the slope efficiency value and decreasing threshold, the figure of merit (overall efficiency) increases. In this work, we have increased this parameter from 57 (for a typical Raman configuration) to 105 (investigated configuration). This represents an $84 \%$ improvement. A threshold of 2.9 watts decreased to 1.3 watts by inserting $1.27 \mathrm{~m}$ of ytterbium doped fiber into a 350-m Raman cavity. Another way for obtaining similar results was explored by enlarging the cavity more than double (to $750 \mathrm{~m}$ ) but power delivery decreased by $20 \%$ due to additional fiber loss. The system studied is shorter and more efficient than typical configurations and might be considered given its simplicity. Advantages, disadvantages and other ways for obtaining similar results are discussed.
\end{abstract}

Keywords: - Fiber optics, Raman laser, Raman Amplifiers, Raman converter, Stimulated Raman scattering.

\section{INTRODUCTION}

The necessity for mid-power fiber lasers that effectively pump Tm3+-doped ZBLAN blue-UV emitting (upconversion) fiber lasers [1,2] and 3000-nm fiber lasers based on Ho3+-doped ZBLAN fibers for medical applications [3,4], together with the non-commercial availability of high power lasers in the $1100-1200 \mathrm{~nm}$, motivated our research for a way to further optimize a well known approach that we used before, the Raman fiber laser (RFL). Apart from these motivations, a frequency-doubled version to 550-600 nm would benefit other medical applications and may serve to generate artificial stars that are used as guides for adaptive astronomical telescopes [5-7]. RFL's operate under the Simulated Raman Scattering (SRS) that is a non-linear effect; as a consequence, they require high-pump powers and long fibers to operate. We explored the possibility for providing a stronger signal that produced an earlier operation. Our approach consisted on splicing short lengths of Yb3+-doped fibers to the Raman cavities. The main results are briefly described in this section. Fig. 1(a) contains the results for a 350-m RFL pumped at $1064 \mathrm{~nm}$, the curves fitting the points (black stars and red squares) represent measurements of output (1115-nm stokes) power as function of the pump power. The curve formed by squares corresponds to the typical Raman cavity and the stars to the cavity proposed here with a 1.27$\mathrm{m}$ spliced section of $\mathrm{Yb3}$ +-doped fiber. Note the clear enhancement of the operating threshold that decreased from $2.9 \mathrm{~W}$ to $1.3 \mathrm{~W}$. Fig. 1(b) shows a zooming of the dashed rectangle of Fig. 1(a), it also includes residual pump signal and both curves are formed by stars. The level of coupled pump at which the stokes-level equals the residual pump (i.e., the critical power) is $\sim 1.8$ watts and each delivered signal has $\sim 0.75$ watts (see the vertical and horizontal dashed-black lines). A numerical modeling for a non-doped cavity that would have the same critical power is represented by the solid curves (red in color view); this cavity would have a length of 755 meters meaning that additional $405 \mathrm{~m}$ of fiber are necessary to equal the critical power of the cavity containing the doped fiber. Most important, the longer cavity delivers less power, 0.6 watts each component (horizontal red-dashed line). Then, for the same critical power, the doped version delivers $1.5 \mathrm{~W}$ that compared to $1.2 \mathrm{~W}$ of the non-doped one represents $25 \%$ more power. Accounting for conversion efficiencies, $(1.2 / 1.8=0.666) \sim 67 \%$ corresponds to the non-doped and $(1.5 / 1.8=0.8333) \sim 83 \%$ to the doped version. This represents a $\sim 16 \%$ efficiency enhancement. Here total efficiency is important because RFL's usually convert all the pump into stokes signal. Apart from the amplified spontaneous emission (ASE) provided by the doped fiber that triggers the laser earlier, this notorious improvement results from the lower loss of the sorter cavity. On the base of these results one may affirm that $\sim 1$ meter of Yb-doped fiber saves hundreds of meters of non-doped Raman fiber. In addition to this, the enhanced system delivers more power. 

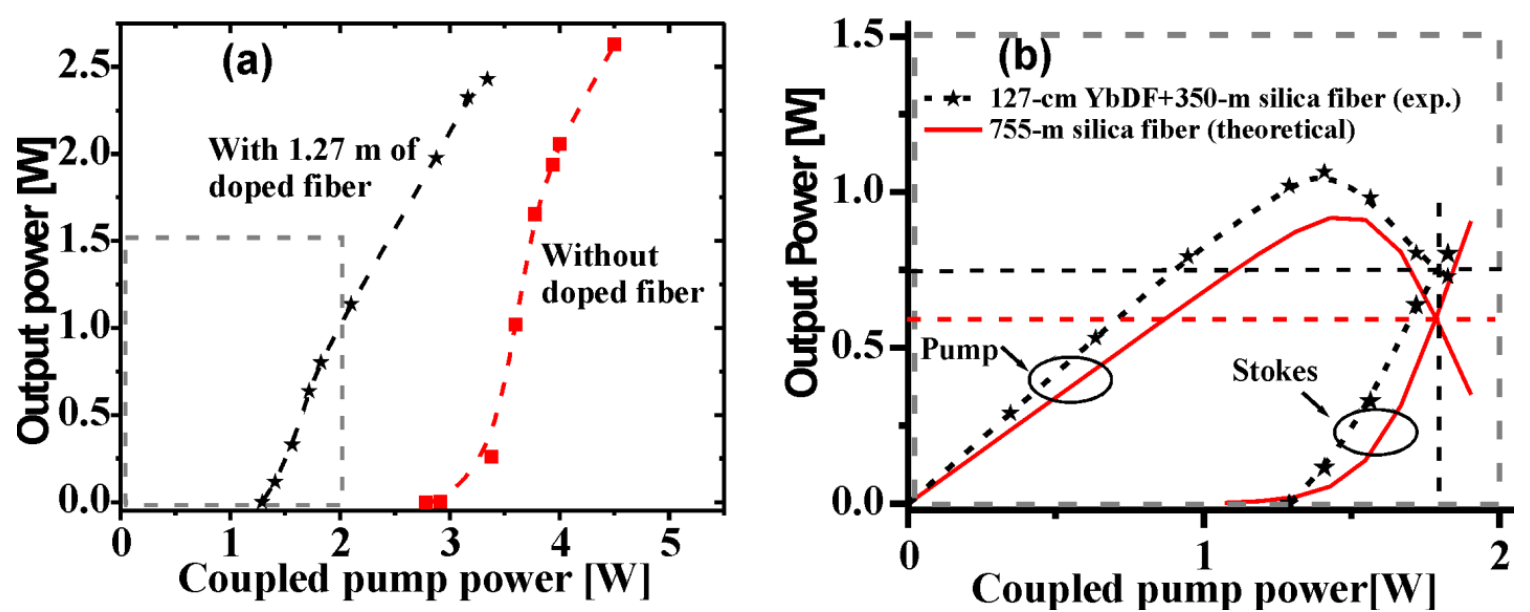

Fig. 1. (a) Dependency of output power on pump power for a 350-m non-doped Raman laser cavity (squares) and for the same cavity with 1.27-m ytterbium-doped fiber (stars); (b) zooming of the dashed rectangle of part

(a) with residual pump included (stars) and modeling (solid curve) of a 755-m non-doped (typical) cavity.

\section{PRINCIPLES OF RAMAN FIBER LASERS}

An intense optical beam coupled into the core of a silica fiber changes its optical properties by the SRS effect. Each atom within a glass molecule has a unique set of vibrating frequencies (modes) that can be taken according to its temperature. Apart from atomic differences, the bonding among them produces frequency bands (also called molecular bands). At temperatures close to $300 \mathrm{~K}$ and in thermal equilibrium, most atoms inside the molecules vibrate at certain mode; let us say that it is located at the lowest energy level shown in Fig. 2(a) with molecular density NO. In silica glass, the remaining molecules are distributed among the other modes that form a band with density population $\mathrm{N} 1$ whose frequency peak is $13.2 \mathrm{THz}$ higher than the corresponding to N0. N1 increases at higher temperatures. The quantum of energy released by an N1 molecule that makes a transition to N0 is the phonon represented by the dotted arrow in the Fig. 2.

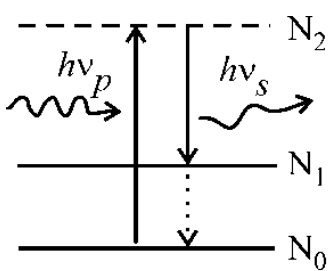

(a)

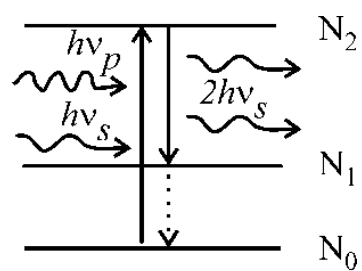

(b)

Fig. 2. (a) Raman scattering (b) stimulated Raman scattering.

When pumping with a weak optical beam the glass, most molecules behave as electric dipoles that harmonically follow the (sinusoidal) electric field acquiring the pump photon energy hvp and become N2 that occupies a virtual level. This level does not correspond to vibration; it is the polarizability responsible of light propagation in transparent media. However, the few atoms vibrating at N1 modulate a small portion, typically 10-6 of the pump beam, creating two bands [8]. In one of them, the phonons add on energy to the photons while in the other they take off energy. The latter is much more efficient and produces stokes photons (hvs). This light-matter interaction is elastic because the optical properties of the glass are not changed by the applied beam. This is the spontaneous Raman scattering (RS) produced by the pump-independent randomly-oriented molecular vibrations that are non-spatially oriented and continuously oscillate in a non-coherent way, i.e., they are not oscillating in phase.

In an optical fiber however, a small portion of the isotropic emitted RS becomes guided in the core and co-propagates with the pump. Further increasing the pump intensity at levels at which the molecules are not able to harmonically follow the electric field, apart from increasing the level of RS signal, a non-elastic interaction occurs. The RS signal triggers a phonon-assisted stimulated emission. This stokes amplification process maintains itself by annihilating pump photons as follows. The sinusoidal shape of the pump electric field (supposing it is monochromatic) becomes distorted, implying the tendency of the electric dipoles to redistribute the pump photons into new frequency-shifted ones that are coherently-driven and time-delayed $[9,10]$. In particular, the ones that coincide with the stokes become around 1-ps delayed; this implies energy accumulation on the virtual level that now, as in lasers, is metastable. From this level, the resonating RS signal triggers an 
avalanche process of stimulated transitions that populate N1 as illustrated in Fig. 2(b) [11]. One RS hvs-photon produces an identical one that propagates in the same direction; these two produce four, and so on. This is the stimulated version of the RS, the stimulated Raman scattering (SRS) that very efficiently produces coherent stokes-photons and phonons. As the metastable level tends to become empty by this process, the pump absorption tends to increase because the photon-phonon generation quickly feeds N0. This is a "virtuous cycle" that annihilates pump photons whereas stokes photons reproduce themselves and phonons dissipate the exhaust energy (heat) through the fiber surface. The stronger the pump, the stronger this cycle with a limit of total energy transfer from pump to stokes. This makes the efficiency of RFL's superior to typical lasers because all the molecules within the volume are active. More than 90\% pump absorption in RFL's is considered typical and most of it converts into stokes signal that may become the pump that generates a second stokes in a longer fiber. This phenomenology supports the development of RFL's and Raman fiber amplifiers (RFA's) [11].

The simplest RFL system consists on pumping an optical fiber (free-running). When pumping some hundreds of meters of standard single-mode silica fiber with few watts of 1064-nm at most two stokes components are generated. It is convenient to introduce a narrow-band high-reflecting mirror at the pumping end that reinforces only a small portion of the RS spectrum. The system is not free-running anymore and the airglass interface at the output completes the resonant cavity; in this way the efficiency increases, the fiber required is shorter and the signal is delivered through a single end. This single-line emitting RFL is modified to multiline emission by adding mirrors for other wavelengths. For this purpose, fiber Bragg gratings (FBG's) are usually spliced to the Raman amplifying fiber (RAF) [12]. A notorious improvement of these systems, as we propose in this work, consists on splicing pieces of Yb3+-doped fiber (YbDF) to the RAF. In such scheme, the YbDF produces in-axis broad-band amplified spontaneous emission (ASE) that ranges from the pump wavelength to around $1160 \mathrm{~nm}[7,13]$. The fiber used here has a core of $4.2 \mu \mathrm{m}$ in diameter and $10000 \mathrm{ppmwt}$ concentration of $\mathrm{Yb}$.

\section{EXPERIMENTS AND RESULTS}

The RAF chosen for our experiments was a single-mode (SM), silica fiber (Nufern 980-HP) whose loss spectrum was obtained by the cut-back technique [14], obtaining $\sim 2.2 \mathrm{~dB} / \mathrm{km}$ at pump (1064 nm), 1.86 at the first stokes $(1115 \mathrm{~nm})$ and $\sim 1.6$ at the second $(1175 \mathrm{~nm})$. By measuring the intensity of the first stokes delivered by a $1.5-\mathrm{km}$ fiber in a free-running configuration, the estimated Raman gain coefficient $(\mathrm{gR})$ was $1.6 \mathrm{dBkm}^{-1} \mathrm{~W}^{-}$ ${ }^{1}$. These parameters introduced in the propagation equations give the required fiber length $(350 \mathrm{~m})$ that generates (weak) first-stokes for 4.5-W coupled pump. The configurations that we characterized are: (a) free-running (Fig. 3), (b) cascaded (Fig. 4), and (c) cascaded-ASE (Fig. 5). In all configurations the output end of the fiber was flat ( $\sim 4 \%$ reflection) and in the latter two the FBG's were patterned on Flexcor-1060 fiber with core diameter of $\sim 6$ $\mu \mathrm{m}$. The transmission spectrum of FBG1 and FBG2 whose maximum reflectance coincide with the first and second stokes respectively is shown in the inset of the Fig. 5. The YbDF and the 980-HP fiber have the same core diameters and hence the loss of the splice is negligible. An optically isolated CW 1064-nm (with less than 1 $\mathrm{nm}$ bandwidth) laser was spliced to each configuration with splicing losses of $0.45 \mathrm{~dB}$ and the splices to the 980-HP fiber were about $1.94 \mathrm{~dB}$. Then, a total loss of $2.34 \mathrm{~dB}$ limited the maximum pump power that could be coupled into the scheme of Fig. 5.

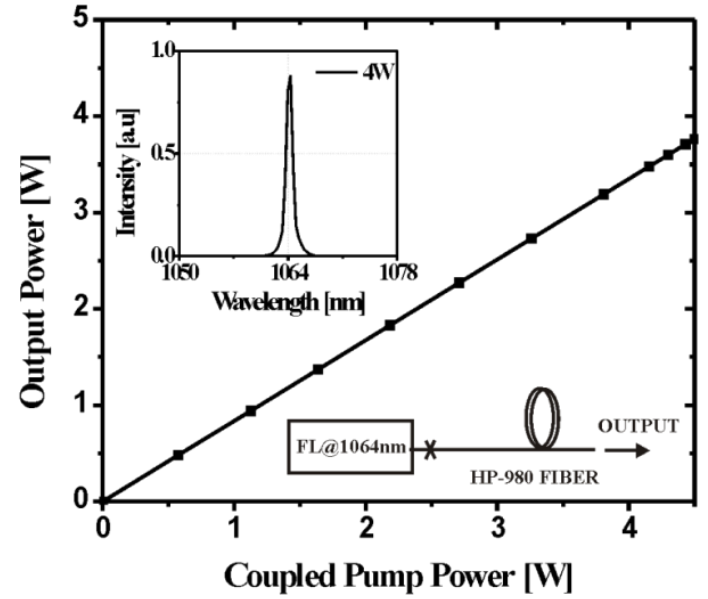

Fig. 3. Output power as a function of coupled power in the free running configuration. The inset shows the output spectrum at $4 \mathrm{~W}$ of pump power.

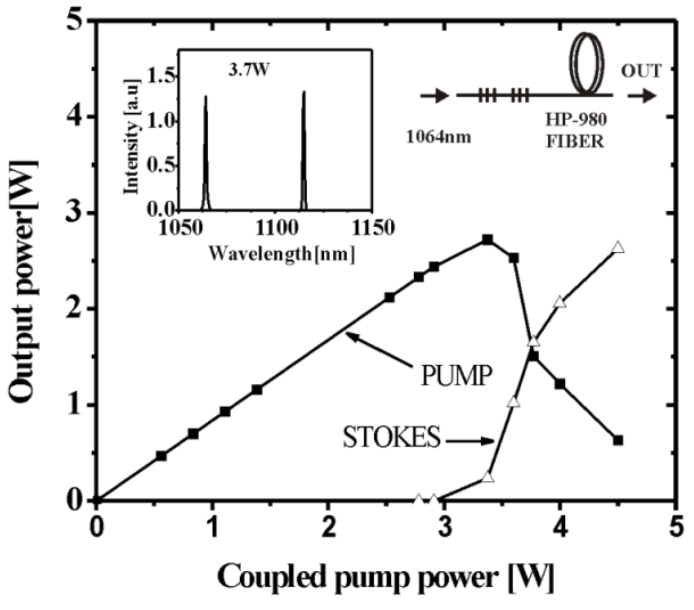

Fig. 4. Output power versus coupled power in the cascaded configuration. The inset shows the output spectrum at $3.7 \mathrm{~W}$ of pump power. 
In the free running configuration, the 350-m silica fiber alone was used as the Raman gain medium and the Fig. 3 shows the output power versus the pump power (see the spectrum in the inset for 4 watts). The first stokes (not shown) just appeared at $\sim 4.5 \mathrm{~W}$. The difference between the output power and the coupled power corresponds to the propagation loss $(2.2 \mathrm{~dB} / \mathrm{km})$. For the cascaded configuration (Fig. 4), the first stokes generation threshold was $\sim 2.9 \mathrm{~W}$. As a reference, the pump-to-stokes conversion efficiency at $4 \mathrm{~W}$ is $\sim 52 \%$ and the ratio of slope efficiency to threshold pump (i.e. the figure of merit as defined in [15]) is 57. The curves composed of squares and triangles represent respectively, the residual pump and the first stokes delivered signals, whereas the inset shows the spectrum for $3.7 \mathrm{~W}$-pump, each signal was 1.6 watts with bandwidths of 0.9 and $0.88 \mathrm{~nm}$.

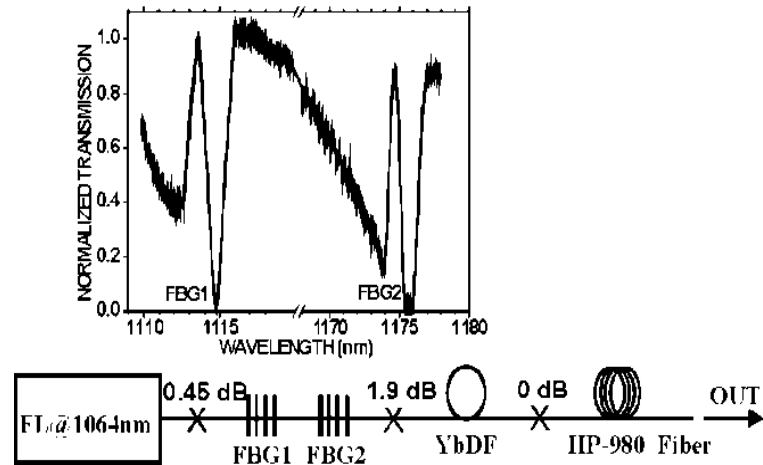

Fig. 5. Scheme of the cascaded-ASE experimental configuration. The inset shows the transmission spectrum of FBG1.

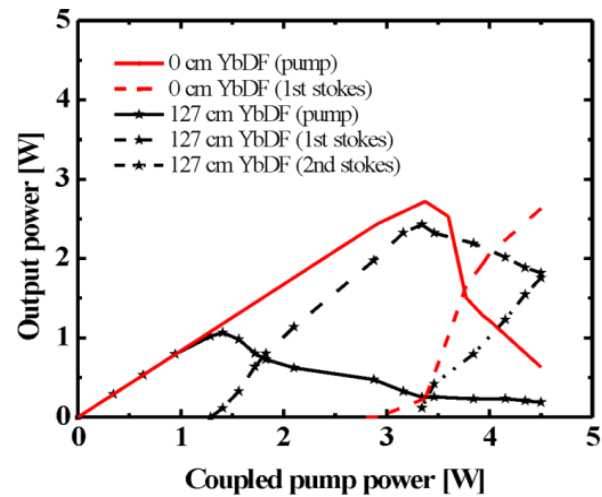

Fig. 6. Output power of the cascaded-ASE configuration. Red curves represent the nondoped configuration and the black ones the 127 cm doped.

As already mentioned, system improvements were achieved by intra-cavity inserting pieces of YbDF as in Fig. 5. Most significant data measurements are presented in Fig. 6 and all signals were CW with their bandwidths dictated by the FBG's. Here, the curves in grey (red in colored figure) are the same of Fig. 4 because they represent the residual pump (solid curve) and stokes (dashes) for 0 - $\mathrm{cm}$ of doped fiber. The curves formed by stars correspond to the cavity with the longer doped fiber $(127 \mathrm{~cm})$. Note that: (a) the non-doped 2.9$\mathrm{W}$ threshold decreased to $1.27 \mathrm{~W}$ for the doped cavity, (b) the pump was almost totally transferred to the first stokes just above $3-\mathrm{W}$, (c) the second stokes starts growing up (and consumes energy from both) at higher levels, (d) the critical power decreased to $1.8 \mathrm{~W}(0.75 \mathrm{~W}$ delivered on each signal), (e) $2.4 \mathrm{~W}$ of maximum stokes was delivered for 3.3-W pump representing $\sim 73 \%$ conversion efficiency and (f) at the $4.5-\mathrm{W}$ critical value for second stokes the total signal converted reaches $3.6 \mathrm{~W}(\sim 1.8 \mathrm{~W}$ each) representing $80 \%$ efficiency. Here, the residual pump $(\sim 200 \mathrm{~mW})$ only represents $\sim 5.6 \%$ of the total delivered whereas the figure of merit (96) represents $68 \%$ improvement. The maximum figure of merit (105) that represents an $84 \%$ improvement was obtained for $55 \mathrm{~cm}$ of YbDF. The complete set of characterizations for all lengths was already reported in [14] and it is briefly summarized in Figs. 7 and 8.

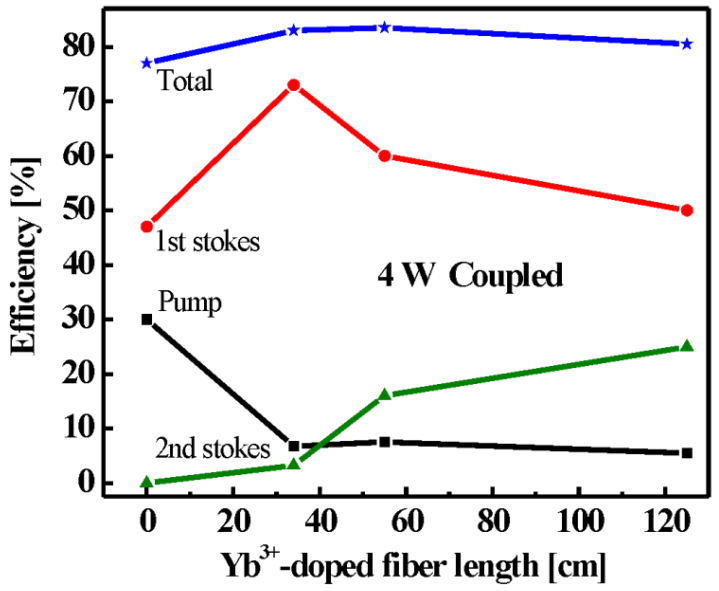

Fig. 7. Efficiencies as function of YbDF length at 4-watt pump. 


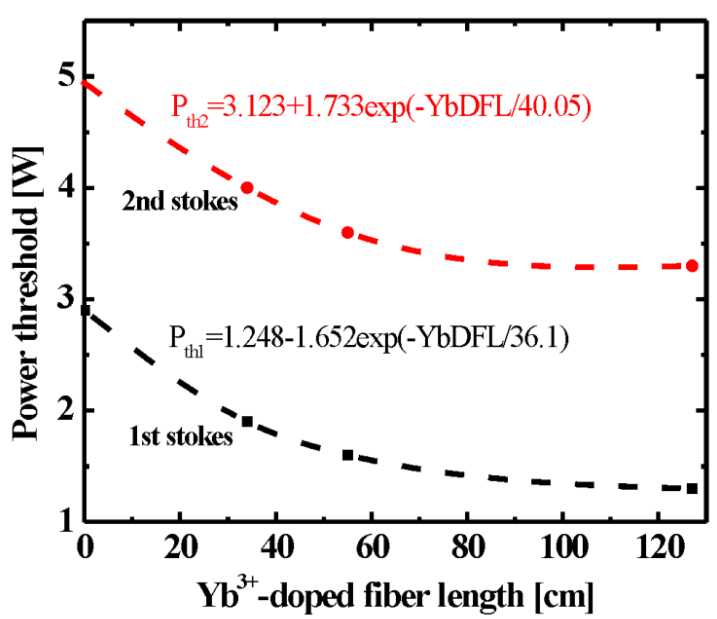

Fig. 8. Power threshold as function of (inserted) doped-fiber length for the 350-m Raman cavity.

Fig. 7 contains the efficiency of each delivered signal as function of doped-fiber length for 4-W coupled pump. The non-doped cavity $(0-\mathrm{cm})$ does not deliver second stokes (first green triangle), delivers $\sim 30$ $\%$ of pump (first black square) and $\sim 7 \%$ of stokes (first red dot) that gives $77 \%$ total efficiency (first black star). An interesting situation takes place for $34 \mathrm{~cm}$ because the second stokes just appears, the pump practically reaches its minimum, $73 \%$ of the power is delivered in the stokes and the total efficiency increases to $\sim 83 \%$. This increase is due to the lower attenuation $(1.86 \mathrm{~dB} / \mathrm{km})$ of the stokes compared to the pump $(2.2 \mathrm{~dB} / \mathrm{km})$. Longer fibers favor energy transfer from first to second stokes. Fig. 8 contains information concerning power threshold for reaching each stokes as function of doped length. The squares (data measurements for first stokes) were fitted by the black-dashed line whose function is Pth1=1.248+1.6525exp(-YbDFL/36.1). Here YbDFL means YbDF-length. Then, the minimum threshold for very long fibers would be $1.248 \mathrm{~W}$ which was almost reached with the longest fiber. The function that corresponds to second stokes (red dashed line with solid circles) is Pth2=3.123+1.733exp(-YbDFL/40.05) and converges to 3.123-W threshold for very long fibers.

An obvious question is why not to simply use an YbDFL to generate $1115 \mathrm{~nm}$. This is a more expensive solution because ten meters are necessary since absorption cross section is quite small. In addition to that, threelevel laser systems are very inefficient given that the whole medium has to be population-inverted resulting in a high level of non-absorbed (residual) pump [16,17]. Then, apart from the higher cost, the efficiency becomes a serious issue. Other approach would be the use a double-clad 976-nm diode-pumped fiber; although the absorption cross section value at this wavelength is quite big, double-clad requires very long fibers to absorb the multi-mode pump. This (also three-level) scheme would require at least 10 meters also with a lower efficiency. Based on the results of this work, we suggest having the dopants more diluted and along all the fiber in order to further improve the system; overcoming in this way, adverse effects related to high concentration such as quenching. An additional enhancement for the second stokes generation is possible by heating the fiber some tens of degrees Celsius because the ASE emission becomes IR-shifted [7]. Other regions may be enhanced by co-doping the system with other rare-earths. Further studies in this direction are under way.

\section{CONCLUSIONS}

The amplified spontaneous emission produced by some RE's as dopants in silica fibers notoriously improve Raman fiber lasers as reported here for $\mathrm{Yb} 3+$. By splicing short pieces of doped fiber inside Raman lasers, pump power thresholds for generating stokes signals decrease and pump absorption increases, making the systems more efficient and shorter. The cost per pumping watt and the high loss to overcome in long fibers are the most important issues to consider in the design of these systems. The scheme presented here contributes to reduce costs because short pieces of doped fiber (as the ones used here) do not cost more than some tens of dollars at present and save hundreds of meters of non-standard silica fiber. We believe that this approach, as others derived from this work such as using other RE's for other wavelengths, will contribute to reduce the cost of these systems.

\section{ACKNOWLEDGEMENTS}

This work was supported by CONACYT grant reference: CB-2011-01/166740 and the UNACAR under Projects DAIT/2013/03. 


\section{REFERENCES}

[1] D.V. Talavera and E.B. Mejia, Blue up-conversion Tm3+-doped fiber laser pumped by a multiline Raman source, Journal of Applied Physics, 97(5), 2005, 053102-053102-4.

[2] E.B. Mejia, Ultraviolet emission in Tm3+-doped fluoride fiber pumped with two infrared wavelengths, Journal of Applied Physics, 100(11), 2006, 113110-113110-4.

[3] D.V. Talavera and E.B. Mejia, Holmium-Doped Fluoride Fiber Laser at $2950 \mathrm{~nm}$ Pumped at $1175 \mathrm{~nm}$, Laser Physics, 16(3), 2006, 436-440.

[4] A.F.H. Librantz, S.D. Jackson, L. Gomes, S.J.L. Ribeiro, and Y. Messaddeq, Pump excited state absorption in holmium-doped fluoride glass, Journal of Applied Physics, 103(2), 2008, 023105-023105-8.

[5] H. Coïc, Analytic modeling of high-gain ytterbium-doped fibre amplifiers, Journal of Optics A: Pure and Applied Optics, 4(2), 2002, 120-129.

[6] C.E. Max, S.S. Olivier, H.W. Friedman, J. An, K. Avicola, B.V. Beeman, H.D. Bissinger, J.M. Brase, G.V. Erbert, D.T. Gavel, K. Kanz, M.C. Liu, B. Macintosh, K.P. Neeb, J. Patience and K.E. Waltjen, Image Improvement from a Sodium-Layer Laser Guide Star Adaptive Optics System, Science, 277(5332), 1997, 1649-1652.

[7] A.S. Kurkov, V.M. Paramonov, and O.I. Medvedkov, Ytterbium fiber laser emitting at $1160 \mathrm{~nm}$, Laser Physics Letters, 3(10), 2006, 503-506.

[8] G.P. Agrawal, Nonlinear Fiber Optics (San Diego, CA; Academic Press, 2007)

[9] S.P. Singh, R. Gangwar, and N. Singh, Nonlinear scattering effects in optical fibers, Progress in electromagnetic research, 74, 2007, 379-405.

[10] D. Hollenbeck and C.D. Cantrell, Multiple-vibrational-mode model for fiber-optic Raman gain spectrum and response function, Journal of the Optical Society of America B, 19(2), 2002, 2886-2892.

[11] J. Bromage, Raman Amplification for Fiber Communications Systems, IEEE Journal of Lightwave Technology, 22(1), 2004, 79-93.

[12] B. Burgoyne, N. Godbout and S. Lacroix, Theoretical analysis of nth-order cascaded continuous-wave Raman fiber lasers. I. Model and resolution, Journal of the Optical Society of America B, 22(4), 2005, 764-771.

[13] H.M. Pask, R. J. Carman, D. C. Hanna, A.C. Tropper, C.J. Mackechnie, P.R. Barber and J.M. Dawes, Ytterbium-Doped Silica Fiber Lasers: Versatile Sources for the 1-1.2 $\mu \mathrm{m}$ Region, IEEE Journal Selected Topics Quantum Electronics, 1(1), 1995, 2-13.

[14] L. de la Cruz-May, and E. B. Mejia, Raman fiber laser improvement by using $\mathrm{Yb}^{3+}$-doped fiber, Laser Physics, 19,(5), 2009, 1017-1020.

[15] A. Bertoni, Analysis of the efficiency of a third order cascaded Raman laser operating at the wavelength of $1.24 \mu \mathrm{m}$, Optical and Quantum Electronics, 29(11), 1997, 1047-1058.

[16] A.E. Siegman, Lasers (Mill Valley, CA; University Science Books, 1986)

[17] E. Mejía-Beltrán, Rare-Earth Doped Optical Fibers, in Y. Moh, W.H. Sulaiman and A. Hamzah (Eds.), Selected Topics on Optical Fiber Technology, (Croatia; InTech 2012) 